\title{
Worldlines and worldsheets for non-abelian lattice field theories: Abelian color fluxes and Abelian color cycles
}

\author{
Christof Gattringer ${ }^{\star}$, Daniel Göschl ${ }^{\star \star}$, Carlotta Marchis ${ }^{\star \star \star}$ \\ ${ }^{1}$ Institut für Physik, Universität Graz, 8010 Graz, Austria
}

\begin{abstract}
We discuss recent developments for exact reformulations of lattice field theories in terms of worldlines and worldsheets. In particular we focus on a strategy which is applicable also to non-abelian theories: traces and matrix/vector products are written as explicit sums over color indices and a dual variable is introduced for each individual term. These dual variables correspond to fluxes in both, space-time and color for matter fields (Abelian color fluxes), or to fluxes in color space around space-time plaquettes for gauge fields (Abelian color cycles). Subsequently all original degrees of freedom, i.e., matter fields and gauge links, can be integrated out. Integrating over complex phases of matter fields gives rise to constraints that enforce conservation of matter flux on all sites. Integrating out phases of gauge fields enforces vanishing combined flux of matter- and gauge degrees of freedom. The constraints give rise to a system of worldlines and worldsheets. Integrating over the factors that are not phases (e.g., radial degrees of freedom or contributions from the Haar measure) generates additional weight factors that together with the constraints implement the full symmetry of the conventional formulation, now in the language of worldlines and worldsheets. We discuss the Abelian color flux and Abelian color cycle strategies for three examples: the SU(2) principal chiral model with chemical potential coupled to two of the Noether charges, SU(2) lattice gauge theory coupled to staggered fermions, as well as full lattice QCD with staggered fermions. For the principal chiral model we present some simulation results that illustrate properties of the worldline dynamics at finite chemical potentials.
\end{abstract}

\section{Introduction}

Dualization techniques have recently received quite some attention in the lattice community as a possible way to solve complex action problems caused by finite density or topological terms (see, e.g., the reviews [1-5]). It is now well established that essentially all abelian bosonic theories with chemical potential can be exactly mapped to dual representations where the partition function is a sum over worldlines and worldsheets, and all weights are real and positive such that Monte Carlo simulations can be done directly in terms of the new degrees of freedom.

For non-abelian theories the program of dualizing the system in terms of worldlines and worldsheets is not very advanced yet. The main reason is the problem of reordering the non-abelian variables of the conventional representation after expansion of the local Boltzmann factor, such that they

\footnotetext{
^Speaker, e-mail: christof.gattringer@uni-graz.at

$\star \star$ e-mail: daniel.goeschl@uni-graz.at

$\star \star \star$ Speaker, e-mail: carla.marchis@uni-graz.at
} 
can be integrated out in closed form. In particular for non-abelian gauge theories the lack of a suitable reordering strategy quickly leads to a proliferation of rather non-local couplings in the resulting dual representation. However, for several (spin-) systems with non-abelian symmetries successful complete dualizations ${ }^{1}$ were discussed recently [1, 6-19]. In all cases a suitable representation was found such that after strong coupling expansion of local Boltzmann factors the original degrees of freedom could be integrated out in closed form. This is a strategy that led to interesting worldline representations for several non-abelian systems and is also the basis for the approach reviewed here.

In our contribution we discuss results for a recently introduced strategy for the dualization of nonabelian theories. In the so-called Abelian color flux and Abelian color cycle approaches one writes all traces and matrix/vector products as sums over color indices (or more generally over 'internal indices') and introduces a dual variable for each individual contribution. At this stage of the dualization the dual variables are simply the expansion indices used for the Taylor series of the individual Boltzmann factors. All terms in the corresponding expansion are complex numbers such that the reordering problem is solved. Using a suitable representation all original degrees of freedom can be integrated out. This leads to a set of constraints that come from integrating out the phases of the original fields. As a result the dual variables are now restricted to a system of worldlines and worldsheets. Integrating the degrees of freedom that are not phases, such as radial degrees of freedom or Haar-measure contributions, leads to weight factors that together with the constraints implement the symmetries of the conventional formulation on the worldline/worldsheet representation.

In order to develop the Abelian color flux and cycle concepts in this paper we discuss them for three different systems: the SU(2) principal chiral model with chemical potentials coupled to two of the conserved charges, the $\mathrm{SU}(2)$ lattice gauge theory coupled to staggered fermions, as well as full QCD with staggered fermions.

\section{The principal chiral model}

The principal chiral model provides a simple example for developing the dualization strategy with Abelian color fluxes outlined in the introduction. It has the advantage that the structure of the action is simpler than in a gauge theory - it consists only of nearest neighbor terms - and thus the idea of the Abelian color flux dualization [20] can be presented in a more transparent way. We couple chemical potentials $\mu_{1}, \mu_{2}$ to two of the conserved charges, which introduces a complex action problem in the conventional approach that is overcome in the dual representation. Furthermore the chemical potentials allow one to monitor how the conserved charges are manifest in the dual representation they turn out to correspond to temporal winding numbers for two species of worldlines.

\subsection{Dualization with Abelian color fluxes}

The conventional degrees of freedom of the model are $\mathrm{SU}(2)$ matrices $U_{x}$ assigned to the sites $x$ of a $d$-dimensional $N^{d-1} \times N_{t}$ lattice with periodic boundary conditions. The action sums traced nearest neighbor terms of the $U_{x}$ over all links $x, v$ of the lattice,

$$
S=-\frac{J}{2} \sum_{x, v}\left(\operatorname{Tr}\left[e^{\delta_{v, d} \sigma_{3} \frac{\mu_{1}+\mu_{2}}{2}} U_{x} e^{\delta_{v, d} \sigma_{3} \frac{\mu_{1}-\mu_{2}}{2}} U_{x+\hat{v}}^{\dagger}\right]+\operatorname{Tr}\left[e^{-\delta_{v, d} \sigma_{3} \frac{\mu_{1}-\mu_{2}}{2}} U_{x}^{\dagger} e^{-\delta_{v, d} \sigma_{3} \frac{\mu_{1}+\mu_{2}}{2}} U_{x+\hat{v}}\right]\right) .
$$

The two chemical potentials $\mu_{1}$ and $\mu_{2}$ give different weights to hops in the forward and backward temporal direction (the direction $v=d$ ). Obviously $S$ has an imaginary part for finite $\mu_{\lambda}, \lambda=1,2$,

\footnotetext{
${ }^{1}$ Complete dualizations in the sense that the full theory was dualized and not only leading terms of a strong coupling series - see however the discussion below.
} 
giving rise to a complex action problem in the conventional form. To obtain the partition function of the model we integrate the Boltzmann factor with a product of Haar measures $\int D U=\prod_{x} \int_{S U(2)} \mathrm{d} U_{x}$ and the partition sum is given by $Z=\int D[U] e^{-S}$.

As already outlined in the introduction, for the Abelian flux representation we write all traces and matrix products as explicit sums over color indices ${ }^{2}$. After some reshuffling of terms the action in the exponent of the Boltzmann factor is written as a double sum over color indices, giving rise to [20]

$$
Z=\int D[U] \exp \left(J \sum_{x, v} \sum_{a, b=1}^{2} M_{v}^{a b} U_{x}^{a b} U_{x+\hat{v}}^{a b^{\star}}\right)=\int D[U] \prod_{x, v} \prod_{a, b=1}^{2} e^{J M_{v}^{a b} U_{x}^{a b} U_{x+\hat{\nu}}^{a{ }^{\star}}},
$$

where we have taken into account the $\mu_{\lambda}$-dependence in the factors $M_{v}^{a b}$ defined as

$$
M_{v}^{11}=e^{\mu_{1} \delta_{v, d}} \quad, \quad M_{v}^{22}=e^{-\mu_{1} \delta_{v, d}} \quad, \quad M_{v}^{12}=e^{\mu_{2} \delta_{v, d}}, \quad M_{v}^{21}=e^{-\mu_{2} \delta_{v, d}} .
$$

In the second step of (2) we have converted the sum in the exponent into a product over Boltzmann factors for the individual terms. Note that in these individual Boltzmann factors only products of complex numbers appear which will allow us to reorder the contributions and organize them according to the sites $x$ where we integrate over the conventional degrees of freedom $U_{x}^{a b}$. In order to access individual entries $U_{x}^{a b}, a, b=1,2$ we use the following explicit representation of the $\mathrm{SU}(2)$ matrices and the Haar measure $\left(\theta_{x} \in[0, \pi / 2], \alpha_{x} \in[-\pi, \pi], \beta_{x} \in[-\pi, \pi]\right)$

$$
U_{x}=\left[\begin{array}{cc}
\cos \theta_{x} e^{i \alpha_{x}} & \sin \theta_{x} e^{i \beta_{x}} \\
-\sin \theta_{x} e^{-i \beta_{x}} & \cos \theta_{x} e^{-i \alpha_{x}}
\end{array}\right] \text { with } \mathrm{d} U_{x}=2 \sin \theta_{x} \cos \theta_{x} \mathrm{~d} \theta_{x} \frac{\mathrm{d} \alpha_{x}}{2 \pi} \frac{\mathrm{d} \beta_{x}}{2 \pi}
$$

The next step is to expand the individual Boltzmann factors for each combination of $x, v, a, b$ into a power series and to reorder the factors according to the sites $x$. The partition sum assumes the form

$$
\begin{aligned}
& Z=\int D[U] \prod_{x, \nu} \prod_{a, b} \sum_{j_{x, \nu}^{a b}=0}^{\infty} \frac{\left(J M_{v}^{a b}\right)^{j_{x, \nu}^{a b}}}{j_{x, \nu}^{a b} !}\left[U_{x}^{a b} U_{x+\hat{\nu}}^{a b^{\star}}\right]^{j_{x, \nu}^{a b}}=\sum_{\{j\}} W_{J, \mu}[j] \int D[U] \prod_{x, \nu} \prod_{a, b}\left[U_{x}^{a b}\right]^{j_{x, \nu}^{a b}}\left[U_{x}^{a b^{\star}}\right]^{j_{x-\hat{v}, \nu}^{a b}} \\
& =\sum_{\{j\}} W_{J, \mu}[j] \prod_{x} 2 \int_{0}^{\frac{\pi}{2}} \mathrm{~d} \theta_{x}\left(\cos \theta_{x}\right)^{1+\sum_{\nu}\left[j_{x, \nu}^{11}+j_{x, \nu}^{22}+j_{x-\hat{v}, \nu}^{11}+j_{x-\hat{v}, \nu}^{22}\right]}\left(\sin \theta_{x}\right)^{1+\sum_{\nu}\left[j_{x, \nu}^{12}+j_{x, \nu}^{21}+j_{x-\hat{v}, \nu}^{12}+j_{x-\hat{v}, \nu}^{21}\right]} \\
& \times \int_{-\pi}^{\pi} \frac{\mathrm{d} \alpha_{x}}{2 \pi} e^{i \alpha_{x} \sum_{\nu}\left[\left(j_{x, y}^{11}-j_{x, y}^{22}\right)-\left(j_{x-\hat{\gamma}, v}^{11}-j_{x-\hat{v}, v}^{22}\right)\right]} \int_{-\pi}^{\pi} \frac{\mathrm{d} \beta_{x}}{2 \pi} e^{i \beta_{x} \sum_{\nu}\left[\left(j_{x, y}^{12}-j_{x, v}^{21}\right)-\left(j_{x-\hat{v}, v}^{12}-j_{x-\hat{v}, v}^{21}\right)\right]} .
\end{aligned}
$$

For each individual Boltzmann factor a summation variable $j_{x, v}^{a b} \in \mathbb{N}_{0}$ was introduced and $\sum_{\{j\}}$ denotes the sum over all configurations of these variables. The weight factor $W_{J, \mu}[j]$ collects all weights generated in the expansion of the Boltzmann factors,

$$
W_{J, \mu}[j] \equiv \prod_{x, v} \prod_{a, b} \frac{\left(J M_{v}^{a b}\right)^{j_{x, v}^{a b}}}{j_{x, v}^{a b} !}=e^{\mu_{1} \sum_{x}\left[j_{x, d}^{11}-j_{x, d}^{22}\right]} e^{\mu_{2} \sum_{x}\left[j_{x, d}^{12}-j_{x, d}^{21}\right]} \prod_{x, v} \prod_{a, b} \frac{J^{j_{x, v}^{a b}}}{j_{x, v}^{a b} !} .
$$

In the last step of (5) we have reordered the factors and collected the terms $U_{x}^{a b}$ for all combinations of $x, a, b$. Subsequently the explicit representation (4) was inserted giving rise to integrals over $\theta_{x}$, $\alpha_{x}$ and $\beta_{x}$ at each site $x$. The integrals over $\alpha_{x}$ and $\beta_{x}$ are Kronecker deltas for the integer valued combinations of the $j_{x, v}^{a b}$ in the respective exponents, and constitute constraints for these combinations

\footnotetext{
${ }^{2}$ Although the site variables $U_{x} \in \mathrm{SU}(2)$ have nothing to do with gluons - still we refer to their indices as "color indices".
} 
at each site $x$. The integrals over $\theta_{x}$ give rise to beta-functions that can be simplified as fractions of factorials, since the constraints imply that the exponents of $\cos \theta_{x}$ and $\sin \theta_{x}$ are odd.

The worldline representation can be simplified further by introducing new variables: the "flux variables" $k_{x, v}^{\lambda} \in \mathbb{Z}, \lambda=1,2$ and the "auxiliary variables" $m_{x, v}^{\lambda} \in \mathbb{N}_{0}, \lambda=1,2$. They are defined as

$$
k_{x, v}^{1}=j_{x, v}^{11}-j_{x, v}^{22}, \quad k_{x, v}^{2}=j_{x, v}^{12}-j_{x, v}^{21}, \quad m_{x, v}^{1}=\frac{j_{x, v}^{11}+j_{x, v}^{22}-\left|k_{x, v}^{1}\right|}{2}, \quad m_{x, v}^{2}=\frac{j_{x, v}^{12}+j_{x, v}^{21}-\left|k_{x, v}^{2}\right|}{2} .
$$

Expressing the $j_{x v}^{a b}$ in terms of the $k_{x, v}^{\lambda}$ and $m_{x, v}^{\lambda}$ one arrives at the final form for the worldline representation of the partition function, which we write as

$$
Z=\sum_{\{k, m\}} W_{J}[k, m] W_{H}[k, m] W_{\mu}[k] \prod_{x} \prod_{\lambda=1}^{2} \delta\left(\nabla k_{x}^{\lambda}\right) .
$$

The partition function is a sum $\sum_{\{k, m\}}$ over all possible configurations of the flux variables $k_{x, v}^{\lambda} \in \mathbb{Z}, \lambda=$ 1,2 and the auxiliary variables $m_{x, v}^{\lambda} \in \mathbb{N}_{0}, \lambda=1,2$, which are both assigned to the links of the lattice. Only the flux variables are subject to constraints, which are implemented as product of Kronecker deltas (we use the notation $\delta(n) \equiv \delta_{n, 0}$ ). They enforce a zero divergence condition for both $k_{x, v}^{1}$ and $k_{x, v}^{2}$ at every site $x$, where the discretized divergence is defined as $\nabla k_{x}^{\lambda} \equiv \sum_{\nu}\left[k_{x, v}^{\lambda}-k_{x-\hat{v}, v}^{\lambda}\right]$. A vanishing divergence implies that at each site $x$ the total flux of $k_{x, v}^{\lambda}$ has to vanish and admissible configurations of the fluxes of $k_{x, v}^{\lambda}, \lambda=1,2$ are closed worldlines for the two species of fluxes.

The admissible configurations of the $k$ - and $m$-variables come with weights which we split into three factors as follows: $W_{J}[k, m]$ collects the factors that were generated when expanding the individual Boltzmann terms. The weight factor $W_{H}[k, m]$ contains the contributions from the $\theta_{x}$-integrations, which gives rise to the beta functions that here simplify to fractions of factorials. Finally $W_{\mu}[k]$ is the term that couples the fluxes to the chemical potentials. The first two weight factors are

$$
W_{J}[k, m]=\prod_{x, v} \prod_{\lambda=1}^{2} \frac{J^{D_{x, v}^{\lambda}}}{\left(D_{x, v}^{\lambda}-m_{x, v}^{\lambda}\right) ! m_{x, v}^{\lambda} !}, W_{H}[k, m]=\prod_{x} \frac{\prod_{\lambda=1}^{2}\left(\frac{1}{2} \sum_{v}\left[D_{x, v}^{\lambda}+D_{x-\hat{v}, v}^{\lambda}\right]\right) !}{\left(1+\frac{1}{2} \sum_{v} \sum_{\lambda}\left[D_{x, v}^{\lambda}+D_{x-\hat{v}, v}^{\lambda}\right]\right) !},
$$

where we use the abbreviation $D_{x, v}^{\lambda} \equiv\left|k_{x, v}^{\lambda}\right|+2 m_{x, v}^{\lambda}$. The weight factor $W_{\mu}[k]$ is given by

$$
W_{\mu}[k]=\prod_{\lambda=1}^{2} \prod_{x} e^{\mu_{\lambda} k_{x, d}^{\lambda}}=\prod_{\lambda=1}^{2} e^{\mu_{\lambda} \sum_{x} k_{x, d}^{\lambda}}=e^{\mu_{1} \beta \omega_{1}[k]} e^{\mu_{2} \beta \omega_{2}[k]} .
$$

In the last step the identity $\sum_{x} k_{x, d}^{\lambda}=N_{t} \omega_{\lambda}[k]$ was used, where $\omega_{\lambda}[k], \lambda=1,2$ are the temporal net winding numbers of the two species of fluxes described by the two sets of dual variables $k_{x, d}^{\lambda}, \lambda=1,2$. The temporal extent $N_{t}$ is the inverse temperature in lattice units and on the right hand side of (10) we replaced $N_{t}$ by the more usual symbol $\beta$.

Let us discuss some properties of the resulting worldline representation: It is obvious that all weight factors are real and positive also for finite $\mu_{\lambda}$. Consequently in the worldline representation (8) the complex action problem is solved and a Monte Carlo simulation is possible in terms of the dual variables. As we have discussed in the previous paragraph, the chemical potentials couple to topological quantities in the dual representation, the temporal winding numbers of the two species of worldlines. This property can also be viewed as a guiding principle for the construction of worldline representations $^{3}$ : Identify a set of Noether charges in the conventional representation and choose a

\footnotetext{
${ }^{3}$ Note that a worldline representation is not necessarily unique - for a different worldline representation of the principal chiral model see [15].
} 


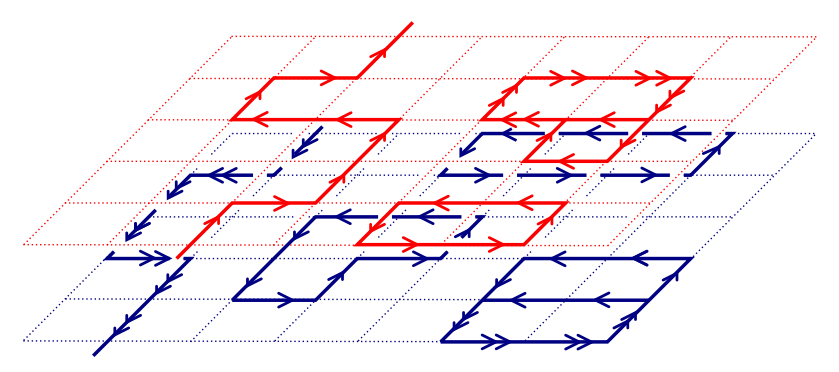

Figure 1: Illustration of the worldline form of the principal chiral model: Admissible configurations consist of two species of worldlines with individually conserved fluxes, which we here illustrate using a space-time lattice (2-dimensional in our illustration) with two layers for the two species of fluxes. On the sites of the lattice the two species of worldlines interact via weight factors that come from the Haar measure integrals and implement the original SU(2) symmetry in the worldline representation.

parameterization such that the corresponding chemical potentials appear with phases, such that after integrating over the conventional degrees of freedom, the chemical potentials couple to temporal winding numbers of worldlines.

The geometrical interpretation of the net-particle numbers as temporal winding numbers implies that for each individual configuration of the dual variables we can determine the net-particle numbers as integers - a property which is not shared by the conventional representation where the net particle number corresponds to a lattice discretization of the continuum Noether charge, which is not necessarily integer. Thus, in the worldline representation it is straightforward to set up a canonical simulation, i.e., a simulation at fixed net-particle number (compare [21]).

Let us finally discuss how the worldline representation implements the SU(2) symmetry of the original representation: The worldlines for the two species of conserved fluxes would correspond to two independent $\mathrm{U}(1)$ charges. However, at each site $x$ of the lattice the two species of worldlines interact with each other and the auxiliary variables via the weight factors $W_{J}[k, m]$. These weights come from integrating over the contributions containing the angles $\theta_{x}$ with the Haar measure, thus implementing the SU(2) symmetry of the conventional representation. A graphical illustration of the emerging geometrical picture is shown in Fig. 1.

\subsection{Monte Carlo simulation and some results}

The dual representation of the principal chiral model (8) does not exhibit a sign problem at finite $\mu_{\lambda}$ and a Monte Carlo simulation is possible in terms of the worldlines. In this brief explorative 4-d study we consider bulk observables, which can be obtained as derivatives of the logarithm of the partition sum. More specifically, we investigate the action density $\langle s\rangle$ and the particle number densities $\left\langle n_{\lambda}\right\rangle$, as well as the action density susceptibility $\chi_{s}$ and the particle number susceptibilities $\chi_{n_{\lambda}}$ defined as

$$
\langle s\rangle=\frac{1}{8 V} \frac{\partial}{\partial J} \ln (Z), \quad \chi_{s}=\frac{1}{8 V} \frac{\partial^{2}}{\partial J^{2}} \ln (Z), \quad\left\langle n_{\lambda}\right\rangle=\frac{1}{V} \frac{\partial}{\partial \mu_{\lambda}} \ln (Z), \quad \chi_{n_{\lambda}}=\frac{1}{V} \frac{\partial^{2}}{\partial \mu_{\lambda}^{2}} \ln (Z) .
$$

These derivatives can be evaluated also for the dual form and the observables read

$\langle s\rangle=\frac{1}{4 V J}\langle\bar{D}\rangle, \chi_{s}=\frac{1}{4 V J}\left[\left\langle\bar{D}^{2}\right\rangle-\langle\bar{D}\rangle^{2}-\langle\bar{D}\rangle\right],\left\langle n_{\lambda}\right\rangle=\frac{1}{N_{s}^{3}}\left\langle\omega_{\lambda}[k]\right\rangle, \chi_{n_{\lambda}}=\frac{1}{N_{s}^{3}}\left[\left\langle\omega_{\lambda}[k]^{2}\right\rangle-\left\langle\omega_{\lambda}[k]\right\rangle^{2}\right]$, 


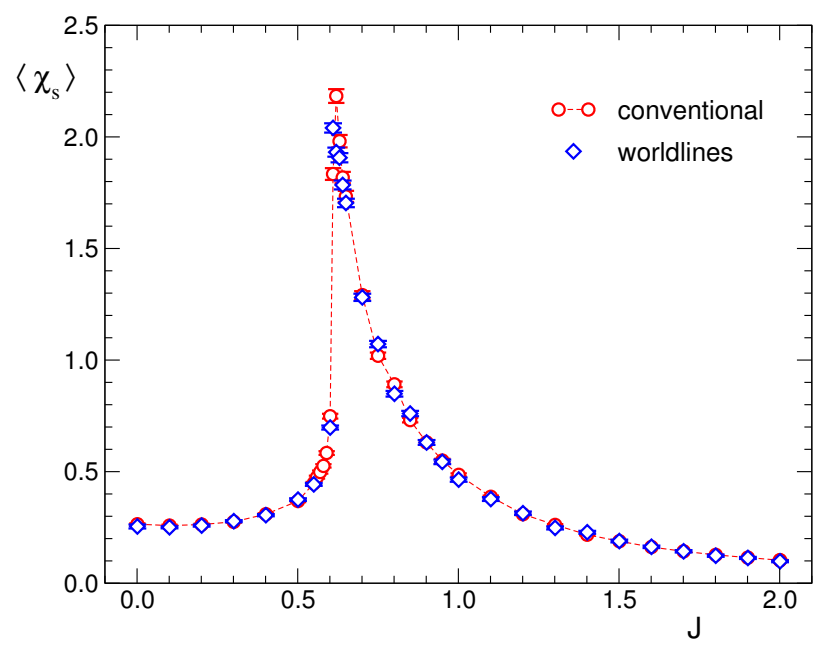

Figure 2: We compare the results for the susceptibility $\chi_{s}$ from the conventional- (circles) and the worldline (diamonds) simulations $\left(16^{4}, \mu_{1}=\mu_{2}=0\right)$.

where we use the shorthand notation $\bar{D}=\sum_{x, v} \sum_{\lambda} D_{x, v}^{\lambda}$.

Our strategy for generating new configurations in the Monte Carlo simulation combines a local Metropolis update for the unconstrained $m$-variables and two separate updates for the $k$-variables which have to obey the constraint of vanishing divergence. For the latter we perform local changes where we offer to change the $k$-flux on the four links of a plaquette by one unit. In addition we explicitly insert winding loops of $k$-flux, in spatial and temporal directions. Both update steps are accepted with a Metropolis decision. The chemical potentials couple to temporally winding loops, giving larger weights to configurations with positive temporal winding numbers $\omega_{\lambda}[k]$. We remark that this update strategy of generating the conserved fluxes from plaquettes and defects (i.e., the winding loops) can be extended to a full Kramers-Wannier dualization where the plaquettes and defect lines are introduced as the new variables [20]. Furthermore we also experimented with a suitable worm strategy [22] for the update but observed severe inefficiencies for large $\beta$ and $\mu$. Possible improvements of the worm update strategy are currently part of our investigations.

In Fig. 2 we show the results for the action density susceptibility $\chi_{s}$ as a function of the coupling $J$ at zero density on a $16^{4}$ lattice. The red circles represent the results obtained from a conventional simulation and the blue diamonds show the results of the worldline simulation performed with the local updates and confirm that the results from the two formulations agree well with each other. For the conventional simulation we used a statistics of $10^{4}$ measurements separated by two heat bath sweeps for decorrelation per coupling, after equilibrating with $10^{3}$ heat bath sweeps. In the worldline simulation we used for each coupling $10^{4}$ equilibration sweeps and a statistics of $2.5 \times 10^{4}$ measurements, separated by 5 decorrelation steps.

After verifying the worldline simulation at $\mu_{\lambda}=0$, we investigate the dynamics of the system at finite density. In Fig. 3 we show time series of the winding numbers as a function of the Monte Carlo time $t$, after an initial thermalization with $10^{3}$ update sweeps. We performed runs on a $16^{3} \times 4$ $\left(N_{t}=4\right)$ lattice at a fixed coupling of $J=0.1$ and for three different chemical potentials $\mu$, where we look at two different scenarios: $\mu_{1}=\mu_{2}=\mu$ and $\mu_{1}=\mu, \mu_{2}=0$. Obviously the time series do 


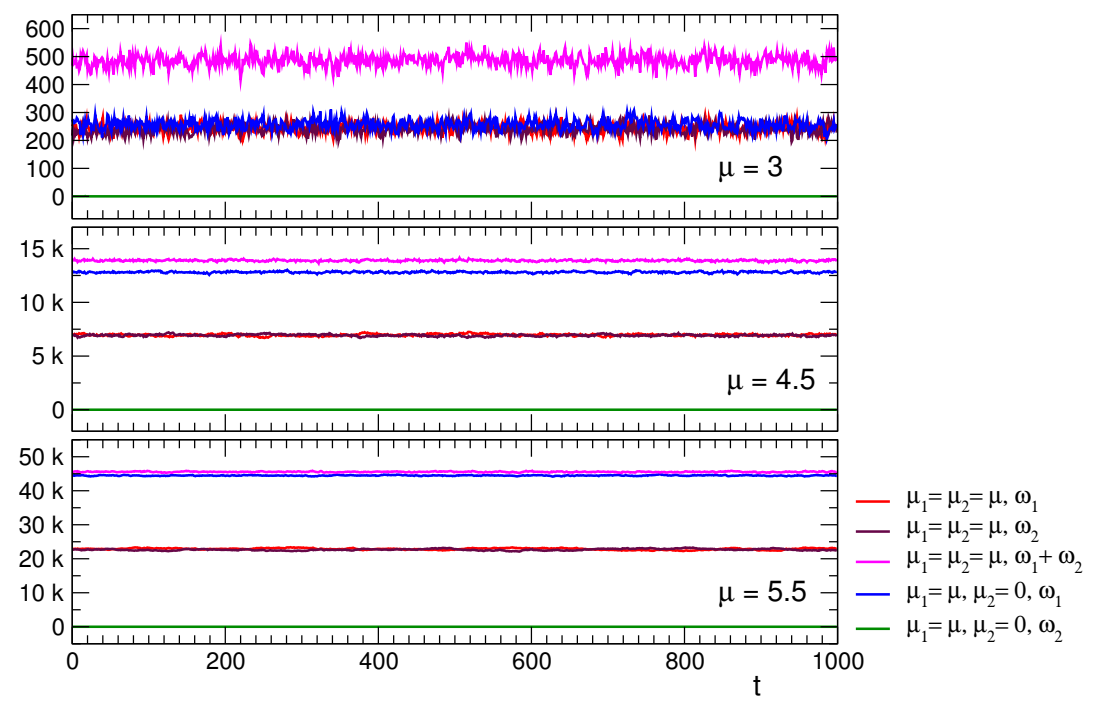

Figure 3: Time series for the temporal winding numbers $\omega_{\lambda}$ (which correspond to the particle numbers) on $16^{3} \times 4$ lattices at $J=0.1$. We analyze two different scenarios: $\mu_{1}=\mu_{2}=\mu$ and $\mu_{1}=\mu$, $\mu_{2}=0$ for three different values of the parameter $\mu$.

not display long autocorrelations for either scenario. The red and maroon lines represent the winding numbers $\omega_{1}$ and $\omega_{2}$ for the first scenario. As expected, they coincide for all three values of $\mu$ due to the equality of the chemical potentials. The blue and green lines represent the two winding numbers for the second scenario. Since in that case $\mu_{2}=0$, also the winding number of the second flavor $\omega_{2}$ vanishes, while $\omega_{1}$ has a non-vanishing value. The interesting question is whether the system favors a certain total winding number. In Fig. 3 we show the combination $\omega_{1}+\omega_{2}$ for the first scenario with a pink line. Comparing the plots, we see that for increasing $\mu$ the relative deviation of the total flux in the two scenarios decreases which indicates that the site couplings of the worldlines coming from the Haar measure integration strongly ties together the two individual fluxes and generates a coherent dynamics of all worldline degrees of freedom (compare the schematic illustration in Fig. 1).

\section{Dual formulation for SU(2) gauge theories}

Having presented the idea of dualization with abelian color fluxes for the SU(2) principal chiral model, the generalization of the idea to $\mathrm{SU}(2)$ lattice gauge theory is more or less straightforward [23, 24]. We discuss the dualization in two steps, first for pure $\mathrm{SU}(2)$ lattice gauge theory and then for the case of $\mathrm{SU}(2)$ gauge fields coupled to staggered fermions.

\subsection{Dual formulation for pure SU(2) lattice gauge theory with abelian color cycles}

The degrees of freedom of SU(2) lattice gauge theory are the link variables $U_{x, \mu} \in \mathrm{SU}(2)$, assigned to the links of the lattice. The partition function is given by $Z=\int D[U] e^{-S_{G}[U]}$, where the path integral measure is the product of the invariant Haar measures $\int D[U]=\prod_{x, \mu} \int_{\mathrm{SU}(2)} d U_{x, \mu}$ over all links. For 


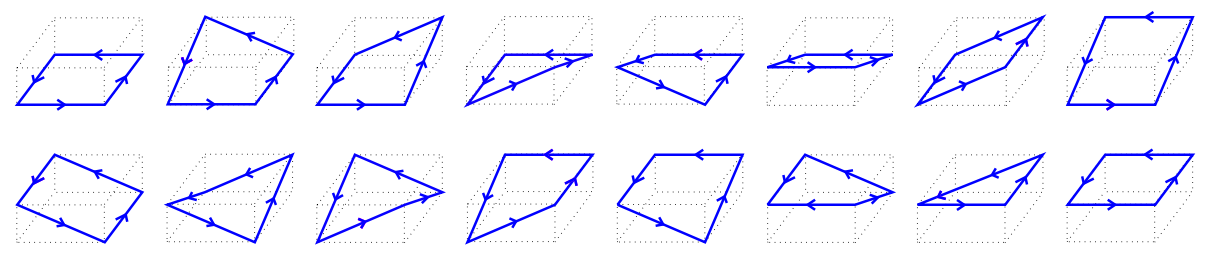

Figure 4: The 16 abelian color cycles of SU(2): The ACCs are paths in color space closing around a plaquette. In our graphical representation the lower layer corresponds to color index 1, while the upper layer is color index 2. At each site of a plaquette the ACC can run through color 1 or 2 giving rise to the 16 different ACCs we show.

the gauge action we use the Wilson form,

$$
S_{G}[U]=-\frac{\beta}{2} \sum_{x, \mu<v} \operatorname{Tr} U_{x, \mu} U_{x+\hat{\mu}, v} U_{x+\hat{v}, \mu}^{\dagger} U_{x, v}^{\dagger}=-\frac{\beta}{2} \sum_{x, \mu<v} \sum_{a, b, c, d=1}^{2} U_{x, \mu}^{a b} U_{x+\hat{\mu}, v}^{b c} U_{x+\hat{v}, \mu}^{d c \star} U_{x, v}^{a d} \text { *, }
$$

where in the second step we have already rewritten the trace and the matrix products as explicit sums over the SU(2) color indices. The partition function is then written as

$$
\begin{gathered}
Z=\int D[U] \prod_{x, \mu<v} \prod_{a, b, c, d=1}^{2} e^{\frac{\beta}{2} U_{x, \mu}^{a b} U_{x+\hat{\mu}, v}^{b c} U_{x+\hat{\nu}, \mu}^{d c} U_{x, \nu}^{a d} \star} \\
=\int D[U] \prod_{x, \mu<v} \prod_{a, b, c, d=1}^{2} \sum_{p_{x, \mu \nu}^{a b c d}=0}^{\infty} \frac{(\beta / 2)^{p_{x, \mu \nu}^{a b c d}}}{p_{x, \mu \nu}^{a b c d} !}\left(U_{x, \mu}^{a b} U_{x+\hat{\mu}, \nu}^{b c} U_{x+\hat{v}, \mu}^{d c \star} U_{x, \nu}^{a d \star}\right)^{p_{x, \mu \nu}^{a b c d}},
\end{gathered}
$$

where in the first step the exponential of the sum was rewritten as a product of individual exponentials. In the second step each of these individual exponentials was expanded in its Taylor series. The corresponding expansion indices $p_{x, \mu v}^{a b c d} \in \mathbb{N}_{0}$ appear as powers of the product $U_{x, \mu}^{a b} U_{x+\hat{\mu}, v}^{b c} U_{x+\hat{v}, \mu}^{d c \star} U_{x, v}^{a d} \star$ of elements of the link variables. This product corresponds to a path in color space closing around a plaquette and is referred to as abelian color cycle (ACC). The indices $p_{x, \mu \nu}^{a b c d}$ we refer to as "cycle occupation numbers". For SU(2) there are 16 different color cycles which are distinguished by the possible combinations of the color indices $a, b, c, d \in\{1,2\}$. In Fig. 4 we illustrate these 16 ACCs.

The ACCs $U_{x, \mu}^{a b} U_{x+\hat{\mu}, v}^{b c} U_{x+\hat{v}, \mu}^{d c \star} U_{x, v}^{a d}$ are complex numbers, such that we can reorder them in (14) and organize them with respect to the individual links where they are integrated over with the Haar measure. Introducing the notation $\sum_{\{p\}}=\prod_{x, \mu<v} \prod_{a, b, c, d=1}^{2} \sum_{p_{x, \mu \nu}^{a b c d}=0}^{\infty}$ for the sum over all configurations of the cycle occupation numbers $p_{x, \mu \nu}^{a b c d} \in \mathbb{N}_{0}$ and reordering the ACCs we find for the partition sum

$$
Z=\sum_{\{p\}}\left[\prod_{x, \mu<v} \prod_{a, b, c, d} \frac{(\beta / 2)^{p_{x, \mu \nu}^{a b c d}}}{p_{x, \mu \nu}^{a b c d} !}\right] \prod_{x, \mu} \int d U_{x, \mu} \prod_{a, b}\left(U_{x, \mu}^{a b}\right)^{N_{x, \mu}^{a b}}\left(U_{x, \mu}^{a b \star}\right)^{\bar{N}_{x, \mu}^{a b}}
$$

where we collected the exponents $N_{x, \mu}^{a b}$ and $\bar{N}_{x, \mu}^{a b}$ for the matrix elements $U_{x, \mu}^{a b}$ and $U_{x, \mu}^{a b}$ given by

$$
N_{x, \mu}^{a b}=\sum_{v: \mu<v} p_{x, \mu v}^{a b s s}+\sum_{\rho: \mu>\rho} p_{x-\hat{\rho}, \rho \mu}^{s a b s} \quad, \quad \bar{N}_{x, \mu}^{a b}=\sum_{v: \mu<v} p_{x-\hat{v}, \mu \nu}^{s s b a}+\sum_{\rho: \mu>\rho} p_{x, \rho \mu}^{a s s b} .
$$




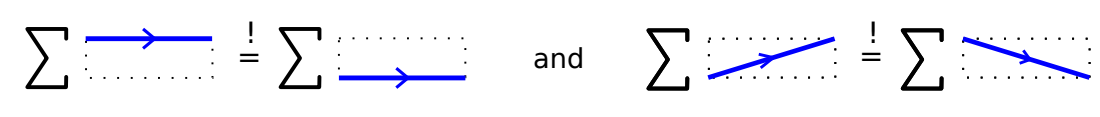

Figure 5: Constraints for the fluxes $J_{x, \mu}^{a b}$ : On all links the sum over all 1-1 flux must be equal to the sum over all 2-2 flux and the sum over 1-2 fluxes must equal the sum over 2-1 fluxes.

The labels $s$ indicate that the corresponding indices are summed independently, for example $p_{x, \mu \nu}^{a b s s} \equiv$ $\sum_{c, d} p_{x, \mu \nu}^{a b c d}$ or $p_{x, \mu \nu}^{s a b s} \equiv \sum_{c, d} p_{x, \mu \nu}^{c a b d}$. In this form the integrals over the gauge link matrix elements $U_{x, \mu}^{a b}$ and $U_{x, \mu}^{a b}$ can now be performed in closed form. Again we use the parameterization (4), but now the $\mathrm{SU}(2)$ matrices are assigned to the links. The partition function turns into

$$
\begin{aligned}
& Z=\sum_{\{p\}}\left[\prod_{x, \mu<v} \prod_{a, b, c, d} \frac{(\beta / 2)^{p_{x, \mu \nu}^{a b c d}}}{p_{x, \mu \nu}^{a b c d} !}\right] \prod_{x, \mu}(-1)^{J_{x, \mu}^{21}} 2 \int_{0}^{\pi / 2} d \theta_{x, \mu}\left(\cos \theta_{x, \mu}\right)^{1+S_{x, \mu}^{11}+S_{x, \mu}^{22}}\left(\sin \theta_{x, \mu}\right)^{1+S_{x, \mu}^{12}+S_{x, \mu}^{21}} \\
& \times \int_{0}^{2 \pi} \frac{d \alpha_{x, \mu}}{2 \pi} e^{i \alpha_{x, \mu}\left[J_{x, \mu}^{11}-J_{x, \mu}^{22}\right]} \int_{0}^{2 \pi} \frac{d \beta_{x, \mu}}{2 \pi} e^{i \beta_{x, \mu}\left[J_{x, \mu}^{12}-J_{x, \mu}^{21}\right]}
\end{aligned}
$$

where we introduced the abbreviations $J_{x, \mu}^{a b}=N_{x, \mu}^{a b}-\bar{N}_{x, \mu}^{a b}$ and $S_{x, \mu}^{a b}=N_{x, \mu}^{a b}+\bar{N}_{x, \mu}^{a b}$. The integrals over the phases $\alpha_{x, \mu}$ and $\beta_{x, \mu}$ give rise to Kronecker deltas which enforce constraints for the fluxes $J_{x, \mu}^{a b}$ at all links $(x, \mu)$. These constraints are

$$
J_{x, \mu}^{11}-J_{x, \mu}^{22}=0 \quad \forall x, \mu \quad \text { and } \quad J_{x, \mu}^{12}-J_{x, \mu}^{21}=0 \quad \forall x, \mu .
$$

As for the case of the SU(2) principal chiral model the constraints imply that the combinations $S_{x, \mu}^{11}+$ $S_{x, \mu}^{22}$ and $S_{x, \mu}^{12}+S_{x, \mu}^{21}$ are even such that we can again write the beta functions from the $\theta_{x, \mu}$-integrations as ratios of factorials.

We obtain the final expression for the dual form of the partition function of SU(2) lattice gauge theory as a sum over configurations of the plaquette occupation numbers $p_{x, \mu v}^{a b c d} \in \mathbb{N}_{0}$ subject to constraints which for a gauge theory are attached to the links of the lattice:

$$
Z=\sum_{\{p\}} W_{\beta}[p] W_{H}[p](-1)^{\sum_{x, \mu} J_{x, \mu}^{21}} \prod_{x, \mu} \delta\left(J_{x, \mu}^{11}-J_{x, \mu}^{22}\right) \delta\left(J_{x, \mu}^{12}-J_{x, \mu}^{21}\right) .
$$

The configurations come with a sign factor that goes back to the parameterization of the SU(2) matrices. Whether it can be absorbed in a suitable resummation of terms in the dual representation is unclear at the moment. We also defined two weight factors

$$
W_{\beta}[p]=\prod_{x, \mu<v} \prod_{a, b, c, d} \frac{\left(\frac{\beta}{2}\right)^{p_{x, \mu \nu}^{a b c d}}}{p_{x, \mu \nu}^{a b c d} !} \quad, \quad W_{H}[p]=\prod_{x, \mu} \frac{\left(\frac{S_{x, \mu}^{11}+S_{x, \mu}^{22}}{2}\right) !\left(\frac{S_{x, \mu}^{12}+S_{x, \mu}^{21}}{2}\right) !}{\left(1+\frac{S_{x, \mu}^{11}+S_{x, \mu}^{22}+S_{x, \mu}^{12}+S_{x, \mu}^{21}}{2}\right) !} .
$$

The $\beta$-dependent weight $W_{\beta}[p]$ collects the trivial factors from the expansion of the exponentials, while the weight factor $W_{H}[p]$ comes from the Haar measure integral and implements the $\mathrm{SU}(2)$ symmetry of the conventional representation in the dual form. 
To conclude this subsection, let us briefly discuss the structure of the constraints, which is interesting for comparison with the SU(3) case we address in the next section. The Kronecker deltas in (19) enforce $J_{x, \mu}^{12}=J_{x, \mu}^{21}$ and $J_{x, \mu}^{11}=J_{x, \mu}^{22} \forall x, \mu$. The fluxes $J_{x, \mu}^{a b}$ collect the contributions of all Abelian color cycles that connect color $a$ at $x$ with color $b$ at $x+\hat{\mu}$. Thus the constraint $J_{x, \mu}^{12}=J_{x, \mu}^{21}$ enforces that along the link $x, \mu$ the flux from color 1 to color 2 is the same as the total flux from color 2 to color 1 . The constraint $J_{x, \mu}^{11}=J_{x, \mu}^{22}$ enforces that along that link the flux between equal colors has to be the same. Thus together the two constraints imply that for both colors the flux is the same for both links, and that along links flux can be exchanged between the two colors. We will find a more general but similar set of constraints for the case of SU(3).

\subsection{Coupling staggered fermions}

Let us briefly discuss the coupling to matter fields and how they can be treated with Abelian color fluxes. More specifically we consider the partition function $Z=\int D[U] e^{-S_{G}[U]} Z_{F}[U]$ where $Z_{F}[U]=$ $\int D[\psi, \bar{\psi}] e^{-S_{F}[\psi, \bar{\psi}, U]}$ is the partition function of staggered fermions in a background of $\mathrm{SU}(2)$ gauge fields. The measure $\int D[\psi, \bar{\psi}]$ is the product measure over Grassmann variables $\psi_{x}^{a}$ and $\bar{\psi}_{x}^{a}$ attached to the sites $x$. The corresponding fermion action is

$$
S_{F}[\bar{\psi}, \psi, U]=\sum_{x}\left[m \sum_{a} \bar{\psi}_{x}^{a} \psi_{x}^{a}+\sum_{\mu} \frac{\gamma_{x, \mu}}{2} \sum_{a, b}\left(\bar{\psi}_{x}^{a} U_{x, \mu}^{a b} \psi_{x+\hat{\mu}}^{b}-\bar{\psi}_{x+\hat{\mu}}^{b} U_{x, \mu}^{a b \star} \psi_{x}^{a}\right)\right],
$$

where $\gamma_{x, \mu}$ denotes the staggered sign factor and we have written all sums over color indices explicitly. Again we turn all sums in the exponent into products and expand each individual Boltzmann factor:

$$
\begin{gathered}
Z_{F}[U]=\int D[\bar{\psi}, \psi] \prod_{x} \prod_{a} e^{-m \bar{\psi}_{x}^{a} \psi_{x}^{a}} \prod_{x, \mu} \prod_{a, b} e^{-\frac{\gamma_{x, \mu}}{2} \bar{\psi}_{x}^{a} U_{x, \mu}^{a b} \psi_{x+\hat{\mu}}^{b}} e^{\frac{\gamma_{x, \mu}}{2} \bar{\psi}_{x+\hat{\mu}}^{b} U_{x, \mu}^{a b \star} \psi_{x}^{a}} \\
=\int D[\bar{\psi}, \psi] \prod_{x} \prod_{a} \sum_{s_{x}^{a}=0}^{1}\left(-m \bar{\psi}_{x}^{a} \psi_{x}^{a}\right)^{s_{x}^{a}} \prod_{x, \mu} \prod_{a, b} \sum_{k_{x, \mu}^{a b}=0}^{1}\left(-\frac{\gamma_{x, \mu}}{2} \bar{\psi}_{x}^{a} U_{x, \mu}^{a b} \psi_{x+\hat{\mu}}^{b}\right)^{k_{x, \mu}^{a b}} \sum_{\bar{k}_{x, \mu}^{a b}=0}^{1}\left(\frac{\gamma_{x, \mu}}{2} \bar{\psi}_{x+\hat{\mu}}^{b} U_{x, \mu}^{a b \star} \psi_{x}^{a}\right)^{\bar{k}_{x, \mu}^{a b}} \\
=\frac{1}{2^{2 V}} \sum_{\{s, k, \bar{k}\}}(2 m)^{\sum_{x, a} s_{x}^{a}} \prod_{x, \mu} \prod_{a, b}\left(U_{x, \mu}^{a b}\right)^{k_{x, \mu}^{a b}}\left(U_{x, \mu}^{a b \star}\right)^{\bar{k}_{x, \mu}^{a b}}(-1)^{k_{x, \mu}^{a b}}\left(\gamma_{x, \mu}\right)^{k_{x, \mu}^{a b}+\bar{k}_{x, \mu}^{a b}} \\
\quad \times \int D[\bar{\psi}, \psi] \prod_{x} \prod_{a}\left(\bar{\psi}_{x}^{a} \psi_{x}^{a}\right)^{s_{x}^{a}} \prod_{x, \mu} \prod_{a, b}\left(\bar{\psi}_{x}^{a} \psi_{x+\hat{\mu}}^{b}\right)^{k_{x, \mu}^{a b}\left(\bar{\psi}_{x+\hat{\mu}}^{b} \psi_{x}^{a}\right)^{\bar{k}_{x, \mu}^{a b}}}
\end{gathered}
$$

We have introduced summation variables $k_{x, \mu}^{a b}$ for the hopping terms that connect color $a$ at $x$ with color $b$ at $x+\hat{\mu}$ and $\bar{k}_{x, \mu}^{a b}$ for the corresponding inverse hop, as well as variables $s_{x}^{a}$ for the contribution of color $a$ to the mass term. Due to the nilpotency of the Grassmann numbers all these variables can only have the values 0 and 1 and may be viewed as activation numbers for the corresponding terms.

After some reordering of terms we have isolated the remaining Grassmann integral in the last line of (22). This integral depends on the values of the activation numbers and can be $0,-1$ or +1 . A non-zero value is possible only for certain configurations of the activation numbers: The forward and backward hopping terms can form closed loops in both, the space-time lattice and in color space, similar to the Abelian color cycles depicted in Fig. 4. Of course not only plaquettes are possible, but also longer loops, as well as so-called dimers where a hop and its inverse are combined. All loops and dimers come with the corresponding entries $U_{x, \mu}^{a b}$ and $U_{x, \mu}^{a b \star}$ of the link variables along their contour and we refer to this structure as the abelian color fluxes already mentioned before. Finally also with 
the activation numbers $s_{x}^{a}$ for the mass terms we can saturate the Grassmann integral, and we refer to those terms as monomers.

We can summarize the constraint for obtaining a non-zero result for the Grassmann integral as follows: Again we consider the lattice as a product of two space-time lattices, with one lattice for each of the factors. The Grassmann integral is non-zero if each site of that double lattice is either occupied by a monomer, is run through by a loop or is the endpoint of a dimer. For staggered fermions one can also work out the sign for these non-trivial configurations. We do not address the sign determination here and refer to [23] for this step. The factors with the gauge link elements $U_{x, \mu}^{a b}$ and $U_{x, \mu}^{a b \star}$ now have to be combined with the corresponding factors in (15) and are then integrated with $\int D[U]$. Thus the constraints and the weights now also depend on the activation numbers of the fermions. We find the following result for the dual representation of the partition function:

$$
\begin{aligned}
Z & =\frac{1}{2^{2 V}} \sum_{\{p, k, \bar{k}, s\}} C_{F}[s, k, \bar{k}] W_{M}[s] W_{\beta}[p] W_{H}[p, k, \bar{k}] \prod_{x, \mu}(-1)^{J_{x, \mu}^{21}+k_{x, \mu}^{21}+\bar{k}_{x, \mu}^{21}} \prod_{L} \operatorname{sign}(L) \\
& \times \prod_{x, \mu} \delta\left(J_{x, \mu}^{11}+k_{x, \mu}^{11}-\bar{k}_{x, \mu}^{11}-\left[J_{x, \mu}^{22}+k_{x, \mu}^{22}-\bar{k}_{x, \mu}^{22}\right]\right) \delta\left(J_{x, \mu}^{12}+k_{x, \mu}^{12}-\bar{k}_{x, \mu}^{12}-\left[J_{x, \mu}^{21}+k_{x, \mu}^{21}-\bar{k}_{x, \mu}^{21}\right]\right) .
\end{aligned}
$$

The partition function now is a sum over all configurations of the fermion activation numbers $k_{x, \mu}^{a b}$, $\bar{k}_{x, \mu}^{a b}$ and $s_{x}^{a}$, as well as the cycle occupation numbers $p_{x, \mu \nu}^{a b c d}$. The fermion activation numbers come with a fermion constraint function $C_{F}[s, k, \bar{k}]$ which equals 1 for all configurations where each site is either run through by a loop, is the endpoint of a dimer or is occupied by a monomer. Otherwise $C_{F}[s, k, \bar{k}]=0$. The monomer weight function $W_{M}[s]$ collects all the mass term contributions and has the simple form $W_{M}[s]=(2 m)^{\sum_{x, a} s_{x}^{a}}$. The weight factor $W_{\beta}[p]$ remains as in the case of pure gauge theory (Eq. 20), while the weight factor $W_{H}[p, k, \bar{k}]$ from the Haar measure integration now also collects the contributions from the link terms in the fermion action. It still has the form (20), but the arguments $S_{x, \mu}^{a b}$ now also depend on the activation of fermion hops, i.e., $S_{x, \mu}^{a b}=N_{x, \mu}^{a b}+\bar{N}_{x, \mu}^{a b}+k_{x, \mu}^{a b}+\bar{k}_{x, \mu}^{a b}$.

In addition to the signs from the 2-1 components in the SU(2) parameterization (4), also the Grassmann integral contributes to the overall sign. This sign depends only on the loops $L$ of the dual fermions variables and for a loop $L$ is given by $\operatorname{sign}(L)=(-1)^{1+|L| / 2+W_{L}+P_{L}}$. Here $|L|$ is the length of the loop $L, W_{L}$ its temporal winding number, and $P_{L}$ the total number of plaquettes needed to fill the loop with a surface such that $L$ appears as the boundary of the surfaces (see [23] for a detailed discussion of the sign factor). Finally, the constraints take into account also the Abelian color fluxes from the fermion hopping terms, as can be seen by the corresponding activation numbers $k_{x, \mu}^{a b}, \bar{k}_{x, \mu}^{a b}$ in the Kronecker deltas. The constraints are still as illustrated in Fig. 5, but the sums are now over the contributions of all ACCs attached to a link, as well as all fermion fluxes.

The form of the ACC/ACF representation of the partition sum in (23) is very satisfactory from a conceptual point of view. SU(2) lattice gauge theory is exactly represented as a sum over cycle and loop configurations that correspond to closed paths in space time as well as in color. The symmetries of the conventional form appear as constraints for the dual variables and local interaction terms. We remark that one can also couple a chemical potential to the fermions, which as in the case of the principal chiral model would couple to the temporal winding number of the fermion loops, underlining again the highly geometrical nature of the dual representation. However, for a numerical simulation a suitable resummation of negative sign contributions has to be found, as we already remarked in the previous subsection for the case of pure $\mathrm{SU}(2)$ lattice gauge theory. In this context it is interesting to note that the leading terms in the strong coupling series all have positive signs [23] indicating that such a resummation strategy might exist. 


\section{Dual formulation for SU(3) gauge theories}

We conclude this proceedings contribution with a discussion of the challenges and new features that appear when one switches to the gauge group SU(3). Also in this case we start with the derivation of the worldsheet representation for pure SU(3) lattice gauge theory, and then discuss the ACC/ACF dual representation for full QCD.

\subsection{Dual formulation for pure SU(3) lattice gauge theory with abelian color cycles}

The generalization to the SU(3) gauge group of the abelian color cycle (ACC) dualization procedure discussed in Sec. 3.1 starts by noticing that, because of the non pseudo-reality of SU(3), in the Wilson action we have to take the real part of the trace of the plaquette variables explicitly:

$$
S_{G}[U]=-\frac{\beta}{3} \sum_{x, \mu<v} \operatorname{Re} \operatorname{Tr}\left[U_{x, \mu} U_{x+\hat{\mu}, v} U_{x+\hat{v}, \mu}^{\dagger} U_{x, \nu}^{\dagger}\right]=-\frac{\beta}{6} \sum_{x, \mu<v} \sum_{a, b, c, d=1}^{3}\left[U_{x, \mu}^{a b} U_{x+\hat{\mu}, v}^{b c} U_{x+\hat{v}, \mu}^{d c \star} U_{x, v}^{a d \star}+c . c .\right] .
$$

This results in ACCs that can either have mathematically positive $\left(U_{x, \mu}^{a b} U_{x+\hat{\mu}, \nu}^{b c} U_{x+\hat{\nu}, \mu}^{d c} U_{x, v}^{a d \star}\right)$ or negative $\left(U_{x, \mu}^{a b \star} U_{x+\hat{\mu}, v}^{b c \star} U_{x+\hat{\nu}, \mu}^{d c} U_{x, v}^{a d}\right)$ orientation. Both types of ACCs are again complex numbers that may be viewed as cycles along the plaquettes $(x, \mu \nu)$ of the lattice with a path in color space labelled by the indices $a, b, c, d$. For SU(3) each of these indices has 3 possible values such that there is a total of 81 different ACCs. Another consequence of the non pseudo-reality of SU(3) is that when we Taylor expand the locally factorized Boltzmann weight we need two sets of summation variables, $n_{x, \mu \nu}^{a b c d} \in \mathbb{N}_{0}$ and $\bar{n}_{x, \mu \nu}^{a b c d} \in \mathbb{N}_{0}$, that correspond to positive and negative oriented ACCs respectively:

$$
\begin{aligned}
& Z=\int D[U] \prod_{x, \mu<v} \prod_{a, b, c, d=1}^{3} e^{\frac{\beta}{6}\left(U_{x, \mu}^{a b} U_{x+\hat{\mu}, \nu}^{b c} U_{x+\hat{\nu}, \mu}^{d c \star} U_{x, \nu}^{a d \star}\right)} e^{\frac{\beta}{6}\left(U_{x, \mu}^{a b \star} U_{x+\hat{\mu}, \nu}^{b c \star} U_{x+\hat{\nu}, \mu}^{d c} U_{x, \nu}^{a d}\right)} \\
& =\int D[U] \prod_{x, \mu<v} \prod_{a, b, c, d=1}^{3} \sum_{n_{x, \mu \nu}^{a b c d}=0}^{\infty} \sum_{\bar{n}_{x, \mu \nu}^{a b c d}=0}^{\infty} \frac{(\beta / 6)^{n_{x, \mu \nu}^{a b c d}+\bar{n}_{x, \mu \nu}^{a b c d}}}{n_{x, \mu \nu}^{a b c d} ! \bar{n}_{x, \mu \nu}^{a b c d} !}\left(U_{x, \mu}^{a b} U_{x+\hat{\mu}, \nu}^{b c} U_{x+\hat{v}, \mu}^{d c \star} U_{x, \nu}^{a d \star}\right)^{n_{x, \mu \nu}^{a b c d}}(c . c .)^{\bar{n}_{x, \mu \nu}^{a b c d}} \\
& =\sum_{\{n, \bar{n}\}}\left[\prod_{x, \mu<v} \prod_{a, b, c, d} \frac{(\beta / 6)^{n_{x, \mu \nu}^{a b c d}+\bar{n}_{x, \mu \nu}^{a b c d}}}{n_{x, \mu \nu}^{a b c d} ! \bar{n}_{x, \mu \nu}^{a b c d} !}\right] \prod_{x, \mu} \int d U_{x, \mu} \prod_{a, b}\left(U_{x, \mu}^{a b}\right)^{N_{x, \mu}^{a b}}\left(U_{x, \mu}^{a b \star}\right)^{\bar{N}_{x, \mu}^{a b}} .
\end{aligned}
$$

The steps in (25) are analogous to the ones performed in (14) and in (15), but here the integer valued powers $N_{x, \mu \nu}^{a b c d}$ and $\bar{N}_{x, \mu \nu}^{a b c d}$ for the matrix elements $U_{x, \mu}^{a b}$ and $U_{x, \mu}^{a b \star}$ in the last line are

$N_{x, \mu}^{a b}=\sum_{v: \mu<v}\left[n_{x, \mu v}^{a b s s}+\bar{n}_{x-\hat{v}, \mu v}^{s s b a}\right]+\sum_{\rho: \mu>\rho}\left[\bar{n}_{x, \rho \mu}^{a s s b}+n_{x-\hat{\rho}, \rho \mu}^{s a b s}\right], \quad \bar{N}_{x, \mu}^{a b}=\sum_{v: \mu<v}\left[\bar{n}_{x, \mu v}^{a b s s}+n_{x-\hat{v}, \mu v}^{s s b a}\right]+\sum_{\rho: \mu>\rho}\left[n_{x, \rho \mu}^{a s s b}+\bar{n}_{x-\hat{\rho}, \rho \mu}^{s a b s}\right]$,

where again the label $s$ stands for the independent sum over the color indices that are replaced by $s$.

The final form of the partition sum (25) is obtained by choosing an explicit parametrization for the SU(3) matrices [25],

$$
U_{x, \mu}=\left(\begin{array}{ccc}
c_{1} c_{2} e^{i \phi_{1}} & s_{1} e^{i \phi_{3}} & c_{1} s_{2} e^{i \phi_{4}} \\
s_{2} s_{3} e^{-i \phi_{4}-i \phi_{5}}-s_{1} c_{2} c_{3} e^{i \phi_{1}+i \phi_{2}-i \phi_{3}} & c_{1} c_{3} e^{i \phi_{2}} & -c_{2} s_{3} e^{-i \phi_{1}-i \phi_{5}}-s_{1} s_{2} c_{3} e^{i \phi_{2}-i \phi_{3}+i \phi_{4}} \\
-s_{2} c_{3} e^{-i \phi_{2}-i \phi_{4}}-s_{1} c_{2} s_{3} e^{i \phi_{1}-i \phi_{3}+i \phi_{5}} & c_{1} s_{3} e^{i \phi_{5}} & c_{2} c_{3} e^{-i \phi_{1}-i \phi_{2}}-s_{1} s_{2} s_{3} e^{-i \phi_{3}+i \phi_{4}+i \phi_{5}}
\end{array}\right),
$$


where $c_{i}=\cos \theta_{x, \mu}^{(i)}, s_{i}=\sin \theta_{x, \mu}^{(i)}$, with $\theta_{x, \mu}^{(i)} \in[0, \pi / 2]$, and $\phi_{i}=\phi_{x, \mu}^{(i)}$, with $\phi_{x, \mu}^{(i)} \in[-\pi, \pi]$. The normalized Haar measure is $d U_{x, \mu}=1 /\left(2 \pi^{5}\right) d \theta_{1} c_{1}^{3} s_{1} d \theta_{2} c_{2} s_{2} d \theta_{3} c_{3} s_{3} d \phi_{1} d \phi_{2} d \phi_{3} d \phi_{4} d \phi_{5}$. The next step is to insert this expression into the product measure $D[U]=\prod_{x, v} d U_{x, v}$, and the matrix elements $U_{x, v}^{a b}$ into (25). For those matrix elements that are expressed as sums of complex numbers we use the binomial theorem $(x+y)^{N}=\sum_{m=0}^{N}\left(\begin{array}{c}N \\ m\end{array}\right) x^{N-m} y^{m}$, thus introducing new auxiliary variables $m_{x, v}^{a b} \in\left\{0, N_{x, v}^{a b}\right\}$ for the matrix elements $(a, b) \in\{(2,1),(2,3),(3,1),(3,3)\}$ and $\bar{m}_{x, v}^{a b} \in\left\{0, \bar{N}_{x, v}^{a b}\right\}$ for the respective complex conjugate. It is convenient to perform a change of variables,

$$
n_{x, \mu \nu}^{a b c d}-\bar{n}_{x, \mu \nu}^{a b c d}=p_{x, \mu \nu}^{a b c d}, \quad p_{x, \mu \nu}^{a b c d} \in \mathbb{Z} \quad ; \quad n_{x, \mu \nu}^{a b c d}+\bar{n}_{x, \mu \nu}^{a b c d}=\left|p_{x, \mu \nu}^{a b c d}\right|+2 l_{x, \mu \nu}^{a b c d}, \quad l_{x, \mu \nu}^{a b c d} \in \mathbb{N}_{0},
$$

and to introduce the fluxes

$$
\begin{gathered}
J_{x, \mu}^{a b}=\sum_{v: \mu<\nu}\left[p_{x, \mu \nu}^{a b s s}-p_{x-\hat{v}, \mu \nu}^{s s b a}\right]-\sum_{\rho: \mu>\rho}\left[p_{x, \rho \mu}^{a s s b}-p_{x-\hat{\rho}, \rho \mu}^{s a b s}\right] \\
S_{x, \mu}^{a b}=\sum_{v: \mu<\nu}\left[\left|p_{x, \mu \nu}^{a b s s}\right|+\left|p_{x-\hat{v}, \mu \nu}^{s s b a}\right|+2\left(l_{x, \mu \nu}^{a b s s}+l_{x-\hat{v}, \mu \nu}^{s s a}\right)\right]+\sum_{\rho: \mu>\rho}\left[\left|p_{x, \rho \mu}^{a s s b}\right|+\left|p_{x-\hat{\rho}, \rho \mu}^{s a b s}\right|+2\left(l_{x, \rho \mu}^{a s s b}+l_{x-\hat{\rho}, \rho \mu}^{s a b s}\right)\right]
\end{gathered}
$$

At this point the Haar measure integrals can be solved in closed form. In particular, the integrations over the $\theta_{i}$ angles give rise to combinatorial factors, which we collect in the weight factor $W_{H}[p, l, m, \bar{m}]$, whose complete expression will be presented in a forthcoming publication, while integrating the phases $\phi_{i}$ gives rise to constraints $C_{H}[p, m, \bar{m}]$ expressed in terms of Kronecker deltas,

$$
\begin{aligned}
C_{H}[p, m, \bar{m}] & =\prod_{x, \mu} \delta\left(J_{x, \mu}^{11}+J_{x, \mu}^{12}-J_{x, \mu}^{33}-J_{x, \mu}^{23}\right) \delta\left(J_{x, \mu}^{22}+J_{x, \mu}^{12}-J_{x, \mu}^{33}-J_{x, \mu}^{31}\right) \\
& \times \delta\left(J_{x, \mu}^{12}-j_{x, \mu}^{21}-j_{x, \mu}^{23}-j_{x, \mu}^{31}-j_{x, \mu}^{33}\right) \delta\left(J_{x, \mu}^{13}+J_{x, \mu}^{12}-J_{x, \mu}^{31}-J_{x, \mu}^{21}\right) \delta\left(J_{x, \mu}^{32}+J_{x, \mu}^{12}-J_{x, \mu}^{23}-J_{x, \mu}^{21}\right) .
\end{aligned}
$$

We introduced $j_{x, v}^{a b}=m_{x, v}^{a b}-\bar{m}_{x, v}^{a b}$ to simplify the notation. These constraints are graphically represented in Fig. 6. They relate the auxiliary variables $m$ and $\bar{m}$ to the cycle occupation numbers $p$ and enforce relations between combinations of the cycle occupation numbers. They are a generalized form of the constraints for $\mathrm{SU}(2)$ to a lattice with three layers, and again their effect is to restrict the admissible configurations to those that fulfill the flux conservation laws implied by (31).

The final form of the partition function,

$$
Z=\sum_{\{p, l\}} \sum_{\{m, \bar{m}\}} W_{\beta}[p, l] W_{H}[p, l, m, \bar{m}] C_{H}[p, m, \bar{m}](-1)^{\sum_{x, \mu} J_{x, \mu}^{12}+J_{x, \mu}^{23}+J_{x, \mu}^{31}-j_{x, \mu}^{23}-j_{x, \mu}^{31}}
$$

is a sum over the configurations of the new dual variables: the cycle occupation numbers $p_{x, \mu \nu}^{a b c d} \in \mathbb{Z}$, and the auxiliary variables $l_{x, \mu \nu}^{a b c d} \in \mathbb{N}_{0}$ and $m_{x, \mu}^{a b} \in\left\{0, N_{x, \mu}^{a b}\right\}, \bar{m}_{x, \mu}^{a b} \in\left\{0, \bar{N}_{x, \mu}^{a b}\right\}$, with $(a, b) \in$ $\{(2,1),(2,3),(3,1),(3,3)\}$. Admissible configurations have to satisfy the link flux constraints (31). Each configuration comes with the positive weights $W_{\beta}[p, l]$ and $W_{H}[p, l, m, \bar{m}]$, and with sign factors $(-1)^{\sum_{x, \mu} J_{x, \mu}^{12}+J_{x, \mu}^{23}+J_{x, \mu}^{31}-j_{x, \mu}^{2,}-j_{x, \mu}^{31}}$, which origin from the SU(3) parametrization.

\subsection{Dual representation of full lattice QCD}

The coupling of matter fields proceeds in the same way as we discussed for SU(2). We again use the staggered discretization of the fermion action (21), and the dualization of the fermion partition sum reads as in (22), with the only difference that for $S U(3)$ the color labels run from 1 to 3 . The power of the overall factor in front of the sum over the configurations of the fermion dual variables $\{s, k, \bar{k}\}$ 
here is $3 V$ instead of $2 V$. The interpretation of the fermion's dual variables is analogous to the one we gave in Sec. 3.2: $s_{x}^{a}=0,1$ corresponds to the color component $a$ of the mass term on site $x . k_{x, v}^{a b}=0,1$ represents the forward hop from color $a$ to color $b$ on the link $(x, v)$, and $\bar{k}_{x, v}^{a b}=0,1$ is the respective backward hop on the same link.

The Grassmann integral in the last line of (22) is non-vanishing only if each Grassmann variable $\psi_{x}^{a} \bar{\psi}_{x}^{a}$ appears exactly once and we express this condition with the fermion constraint $C_{F}[s, k, \bar{k}]$. As discussed in Sec. 3.2, this constraint can be fulfilled by occupying all the three layers of every site of our lattice with monomers, dimers, or loops. These are the only admissible fermion configurations.

If the constraint $C_{F}[s, k, \bar{k}]$ is satisfied, the Grassmann integral in Eq. (22) gives +1 or -1 . Other signs are generated by the staggered sign factors $\prod_{x, v}\left(\eta_{x, v}\right)^{\sum_{a, b}\left[k_{x, v}^{a b}+\bar{k}_{x, v}^{a b}\right]}$, the activation of forward hops $\prod_{x, v}(-1)^{\sum_{a, b} k_{x, v}^{a b}}$, and the anti-periodic boundary conditions along the time direction. They are the same as for the $\mathrm{SU}(2)$ case and one finds that only loop configurations introduce signs. We can express the sign contribution of every loop $L$ in the same simple form we found for $\operatorname{SU}(2), \operatorname{sign}(L)=$ $(-1)^{1+|L| / 2+P_{L}+W_{L}}$ where $|L|$ is the length of the loop $L, P_{L}$ is the number of plaquettes necessary to cover the surface bounded by the loop $L$, and $W_{L}$ is the number of temporal windings.

After the Grassmann integration the partition sum for QCD reads

$$
Z=\frac{1}{2^{3 V}} \sum_{\{s, k, \bar{k}\}} C_{F}[s, k, \bar{k}] W_{M}[s] W_{\mu}[k, \bar{k}] \prod_{L} \operatorname{sign}(L) \int D[U] e^{S_{G}[U]} \prod_{x, v} \prod_{a, b}\left(U_{x, v}^{a b}\right)^{k_{x, \nu}^{a b}}\left(U_{x, v}^{a b \star}\right)^{\bar{k}_{x, v}^{a b}}
$$

where $W_{M}[s]=(2 m)^{\sum_{x, a} s_{x}^{a}}$ is the monomer weight factor and $W_{\mu}[k, \bar{k}]=e^{\mu \sum_{x} \sum_{a b}\left[k_{x, 4}^{a b}-k_{x, 4}^{a b}\right]}$ is the $\mu$ dependent weight factor. To obtain the final form of $Z$ we only have to perform the Haar integration in (33). This differs from the one discussed in the last section just by the presence of the factors $\prod_{x, v} \prod_{a, b}\left(U_{x, v}^{a b}\right)^{k_{x, v}^{a b}}\left(U_{x, v}^{a b \star}\right)^{\bar{k}_{x, v}^{a b}}$, whose effect modifies the expression of the combinatorial factors collected in the weight $W_{H}[p, l, k, \bar{k}, m, \bar{m}]$ and of the constraints $C_{H}[p, k, \bar{k}, m, \bar{m}]$, but not the procedure to obtain them. In particular, the new weights and the new constraints can be obtained from the ones in the pure gauge case with the substitutions $J_{x, \mu}^{a b} \rightarrow J_{x, \mu}^{a b}+k_{x, v}^{a b}-\bar{k}_{x, v}^{a b}$ and $S_{x, \mu}^{a b} \rightarrow S_{x, \mu}^{a b}+k_{x, v}^{a b}+\bar{k}_{x, v}^{a b}$.

Thus for the dual partition sum of full QCD with staggered fermions we obtain

$$
\begin{gathered}
Z=\frac{1}{2^{3 V}} \sum_{\{p, l\}} \sum_{\{s, k, \bar{k}\}} \sum_{\{m, \bar{m}\}} W_{\beta}[p, l] W_{H}[p, l, k, \bar{k}, m, \bar{m}] W_{M}[s] W_{\mu}[k, \bar{k}] C_{F}[s, k, \bar{k}] C_{H}[p, k, \bar{k}, m, \bar{m}] \\
\times \prod_{L} \operatorname{sign}(L) \prod_{x, v}(-1)^{J_{x, \nu}^{12}+K_{x, v}^{12}+J_{x, v}^{23}+K_{x, v}^{23}+J_{x, v}^{31}+K_{x, v}^{31}-j_{x, v}^{23}-j_{x, v}^{31}},
\end{gathered}
$$

where we use the short hand notation $K_{x, v}^{a b}=k_{x, v}^{a b}-\bar{k}_{x, v}^{a b}$. Eq. (34) is an exact rewriting of the partition function of QCD with staggered fermions in terms of integer valued dual variables: The cycle occupation numbers $p_{x, \mu \nu}^{a b c d} \in \mathbb{Z}$, and the auxiliary variables $l_{x, \mu \nu}^{a b c d} \in \mathbb{N}_{0}, m_{x, \mu}^{a b} \in\left\{0, N_{x, \mu}^{a b}\right\}$ and $\bar{m}_{x, \mu}^{a b} \in\left\{0, \bar{N}_{x, \mu}^{a b}\right\}$, with $(a, b) \in\{(2,1),(2,3),(3,1),(3,3)\}$ represent the gauge degrees of freedom in this formulation, while $s_{x}^{a}, k_{x, v}^{a b}, \bar{k}_{x, v}^{a b} \in\{0,1\}$ are the dual variables for the fermions. In order for a configuration to be admissible, fermions have to saturate the lattice and the gauge constraints $C_{H}[p, k, \bar{k}, m, \bar{m}]$ have to be satisfied. Each configuration comes with a weight given by the $W$ factors in (34), as well as signs, given by the loop and the gauge signs. Also here one needs to find a partial resummation strategy in order to use the dual representation in numerical simulations. 


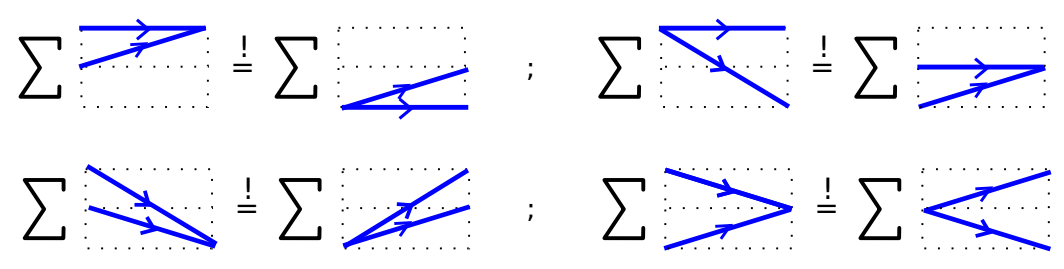

Figure 6: Graphical representation of the constraints for the fluxes in the SU(3) case. The constraints depicted in the first row are a generalization of those for the SU(2) case (compare Fig. 5), as they relate fluxes between different colors. The constraints in the second row enforce flux conservation, since they require that for each link the flux out of a color has to match the flux into that color.

\section{Discussion}

In this contribution we discuss our recent results for the dual representation of lattice field theories with non-abelian symmetries, using the Abelian color cycle / Abelian color flux (ACC/ACF) approaches. We develop the method using three examples: The SU(2) principal chiral model with chemical potentials, as well as $\mathrm{SU}(2)$ and $\mathrm{SU}(3)$ lattice gauge theories with and without fermions.

The key idea of the ACC/ACF approach is to write all matrix/vector products and traces in the action with explicit sums over the color indices. The Boltzmann factor is completely factorized such that only complex numbers remain in the exponent. Subsequently the individual Boltzmann factors are expanded and the resulting factors can be reorganized according to sites or links. Thus the original degrees of freedom can be integrated out with a suitable parameterization. The dual form of the partition function is a sum over closed paths on a lattice which is the product of the space-time lattice with an additional dimension for color. For gauge fields these loops are restricted to plaquettes (ACCs), while for matter fields they can form arbitrary closed paths (ACFs). These worldline configurations are subject to constraints, which enforce conserved combined flux through all links (gauge theories) or all sites (principal chiral model) for each color channel.

For the case of the principal chiral model we could show that all weight factors in the dual representation are real and positive and we present first results of a Monte Carlo simulation of the worldline formulations. For the other models we currently explore the possibility for a partial resummation strategy to overcome the complex action problem.

The dual representations we present here are a variant of the strong coupling expansion where all expansion coefficients are known in closed form. We stress at this point that for QCD there are very interesting recent developments with a differently set up for the strong coupling expansion [26-37] and it would be interesting to better understand the connection between the two versions of the strong coupling series.

Acknowledgments: We thank Falk Bruckmann, Philippe de Forcrand, Mario Giuliani, Yannick Meurice, Oliver Orasch, Tin Sulejmanpasic and Wolfgang Unger for interesting discussions. This work is supported by the FWF DK W 1203, "Hadrons in Vacuum, Nuclei and Stars", and by the FWF project I 2886-N27 in cooperation with DFG TR55, "Hadron Properties from Lattice QCD”.

\section{References}

[1] S. Chandrasekharan, PoS LATTICE2008, 003 (2008), 0810. 2419

[2] P. de Forcrand, PoS LAT2009, 010 (2009), 1005. 0539 
[3] U. Wolff, PoS LATTICE2010, 020 (2010), 1009. 0657

[4] C. Gattringer, PoS LATTICE2013, 002 (2014), 1401. 7788

[5] C. Gattringer, K. Langfeld, Int. J. Mod. Phys. A31, 1643007 (2016), 1603.09517

[6] F. Bruckmann, T. Sulejmanpasic, Phys. Rev. D90, 105010 (2014), 1408. 2229

[7] F. Bruckmann, C. Gattringer, T. Kloiber, T. Sulejmanpasic, Phys. Lett. B749, 495 (2015), [Err.: Phys. Lett. B751 (2015) 595], 1507.04253

[8] F. Bruckmann, C. Gattringer, T. Kloiber, T. Sulejmanpasic, Phys. Rev. Lett. 115, 231601 (2015), 1509.05189

[9] T. Kloiber, C. Gattringer, T. Sulejmanpasic, F. Bruckmann, PoS LATTICE2015, 210 (2016), 1512.05482

[10] F. Bruckmann, C. Gattringer, T. Kloiber, T. Sulejmanpasic, PoS LATTICE2016, 062 (2016), 1611.03228

[11] F. Bruckmann, C. Gattringer, T. Kloiber, T. Sulejmanpasic, Phys. Rev. D94, 114503 (2016), 1607.02457

[12] F. Bruckmann, J. Wellnhofer, PoS LATTICE2016, 054 (2016), 1611.05643

[13] H. Vairinhos, P. de Forcrand, JHEP 12, 038 (2014), 1409.8442

[14] H. Vairinhos, P. de Forcrand, PoS CPOD2014, 061 (2015), 1506.07007

[15] T. Rindlisbacher, P. de Forcrand, PoS LATTICE2015, 171 (2016), 1512.05684

[16] T. Rindlisbacher, P. de Forcrand, Nucl. Phys. B918, 178 (2017), 1610.01435

[17] T. Rindlisbacher, P. de Forcrand, PoS LATTICE2016 (2017), 1703.08571

[18] U. Wolff, Nucl. Phys. B824, 254 (2010), [Err.: Nucl. Phys. B834 (2010) 395], 0908.0284

[19] U. Wolff, Nucl. Phys. B832, 520 (2010), 1001. 2231

[20] C. Gattringer, D. Göschl, C. Marchis (2017), 1709.04691

[21] O. Orasch, C. Gattringer (2017), 1708.02817

[22] M. Giuliani, C. Gattringer (2017), 1702.04771

[23] C. Gattringer, C. Marchis, Nucl. Phys. B916, 627 (2017), 1609.00124

[24] C. Marchis, C. Gattringer, PoS LATTICE2016, 034 (2016), 1611.01022

[25] J.B. Bronzan, Phys. Rev. D38, 1994 (1988)

[26] P. de Forcrand, W. Unger, H. Vairinhos (2017), 1710.00611

[27] P. de Forcrand, P. Romatschke, W. Unger, H. Vairinhos, PoS LATTICE2016, 086 (2017), 1701.08324

[28] J. Kim, W. Unger, PoS LATTICE2016, 035 (2016), 1611.09120

[29] P. de Forcrand, O. Philipsen, W. Unger, PoS CPOD2014, 073 (2015), 1503.08140

[30] W. Unger, PoS LATTICE2014, 192 (2014), 1411. 4493

[31] P. de Forcrand, J. Langelage, O. Philipsen, W. Unger, Phys. Rev. Lett. 113, 152002 (2014), 1406.4397

[32] W. Unger, Acta Phys. Polon. Supp. 7, 127 (2014)

[33] P. de Forcrand, J. Langelage, O. Philipsen, W. Unger, PoS LATTICE2013, 142 (2014), 1312.0589

[34] W. Unger, P. de Forcrand, PoS LATTICE2012, 194 (2012), 1211.7322

[35] M. Fromm, J. Langelage, O. Philipsen, P. de Forcrand, W. Unger, K. Miura, PoS LATTICE2011, 212 (2011), 1111.4677

[36] W. Unger, P. de Forcrand, PoS LATTICE2011, 218 (2011), 1111.1434

[37] W. Unger, P. de Forcrand, J. Phys. G38, 124190 (2011), 1107.1553 